DOI: $10.2478 /$ v10324-012-0004-5<smiles>C1=C2CC[C]1C2</smiles>

VERSITA
Analele Universităţii de Vest,

Timişoara

Seria Matematică - Informatică

L, 1, (2012), 51- 67

\title{
Weighted Lipschitz Estimates for Multilinear Commutator of Integral Operator
}

Du Jing, Huang Chuangxia, and Liu Lanzhe

\begin{abstract}
In this paper, the weighted boundedness for the multilinear commutator associated to some integral operator and the weighted Lipschitz functions are obtained. The integral operator includes the Littlewood-Paley operator, Marcinkiewicz operator and Bochner-Riesz operator.
\end{abstract}

AMS Subject Classification (2000). 42B20, 42B25.

Keywords. Multilinear commutator; Integral operator; Weighted Lipschitz space; Triebel-Lizorkin space; Littlewood-Paley operator; Marcinkiewicz operator; Bochner-Riesz operator.

\section{Introduction}

As the development of the singular integral operators, their commutators and multilinear operators have been well studied. In [8][18-19], the authors proved that the commutators and multilinear operators generated by the singular integral operators and $B M O$ functions are bounded on $L^{p}\left(R^{n}\right)$ for $1<p<$ $\infty$. In [6][14][17], the boundedness for the commutators and multilinear operators generated by the singular integral operators and Lipschitz functions on $L^{p}\left(R^{n}\right)(1<p<\infty)$ and Triebel-Lizorkin spaces are obtained. And the weighted boundedness for the commutators generated by the singular integral operators and $B M O$ or Lipschitz functions on $L^{p}\left(R^{n}\right)(1<p<\infty)$ spaces are obtained(see [3][13]). Inspired by it, we will discuss the weighted boundedness 
for multilinear commutator associated to some integral operators and the weighted Lipschitz functions. The integral operator includes the LittlewoodPaley operator, Marcinkiewicz operator and Bochner-Riesz operator.

\section{Notations and Theorems}

Suppose $b_{j}(j=1, \cdots, m)$ are the fixed locally integrable functions on $R^{n}$. Let $F_{t}(x, y)$ be defined on $R^{n} \times R^{n} \times[0,+\infty)$. Set

$$
F_{t}(f)(x)=\int_{R^{n}} F_{t}(x, y) f(y) d y
$$

and

$$
F_{t}^{\vec{b}}(f)(x)=\int_{R^{n}} \prod_{j=1}^{m}\left(b_{j}(x)-b_{j}(y)\right) F_{t}(x, y) f(y) d y,
$$

for every bounded and compactly supported function $f$. Let $H$ be the Banach space $(H,\|\cdot\|)$ such that, for each fixed $x \in R^{n}, F_{t}(f)(x)$ and $F_{t}^{\vec{b}}(f)(x)$ may be viewed as the mappings from $[0,+\infty)$ to $H$. The multilinear commutator related to $F_{t}$ is defined by

$$
T_{\vec{b}}(f)(x)=\left\|F_{(\cdot)}^{\vec{b}}(f)(x)\right\|,
$$

where $F_{t}$ satisfies: for fixed $\varepsilon>0$

$$
\left\|F_{t}(x, y)\right\| \leq C|x-y|^{-n}
$$

and

$$
\left\|F_{t}(y, x)-F_{t}(z, x)\right\|+\left\|F_{t}(x, y)-F_{t}(x, z)\right\| \leq C|y-z|^{\varepsilon}|x-z|^{-n-\varepsilon},
$$

if $2|y-z| \leq|x-z|$. We also define that $T(f)(x)=\left\|F_{(\cdot)}(f)(x)\right\|$.

Note that when $b_{1}=\cdots=b_{m}, T_{\vec{b}}$ is just the $m$ order commutator (see [1][22]). It is well known that commutators are of great interest in harmonic analysis and have been widely studied by many authors (see[7][9][15][18][19]). Our main purpose is to establish the sharp inequality for the multilinear commutator.

To state our result, we first give some notions(see [1][10][16][19][21]).

In this paper, $Q$ will denote a cube of $R^{n}$ and $f_{Q}=|Q|^{-1} \int_{Q} f(x) d x$, we define the sharp function of $f$ as

$$
f^{\#}(x)=\sup _{Q \ni x} \frac{1}{|Q|} \int_{Q}\left|f(y)-f_{Q}\right| d y .
$$


It is well-known that (see [10])

$$
f^{\#}(x) \approx \sup _{Q \ni x} \inf _{c \in C} \frac{1}{|Q|} \int_{Q}|f(y)-C| d y .
$$

Let $M$ be the Hardy-Littlewood maximal operator defined by

$$
M(f)(x)=\sup _{Q \ni x}|Q|^{-1} \int_{Q}|f(y)| d y .
$$

For $1 \leq p<\infty$ and $0 \leq \eta<n$, we denote $M_{\eta, p}(f)$ by

$$
M_{\eta, p}(f)(x)=\sup _{Q \ni x}\left(\frac{1}{|Q|^{1-p \eta / n}} \int_{Q}|f(y)|^{p} d y\right)^{1 / p} .
$$

The $A_{p}$ weight is defined by (see [10])

$$
\begin{gathered}
A_{p}=\left\{w: \sup _{Q}\left(\frac{1}{|Q|} \int_{Q} w(x) d x\right)\left(\frac{1}{|Q|} \int_{Q} w(x)^{-1 /(p-1)} d x\right)^{p-1}<\infty\right\} \\
1<p<\infty \\
A_{1}=\{w>0: M(w)(x) \leq C w(x), \text { a.e. }\}
\end{gathered}
$$

and $A_{\infty}=\cup_{p \geq 1} A_{p}$. We know, for $w \in A_{1}, w$ satisfies the double condition, that is, for any cube $Q$,

$$
w(2 Q) \leq C w(Q) .
$$

The $A(p, q)$ weight is defined by (see [10])

$$
\begin{gathered}
A(p, q)=\{w>0: \\
\left.\sup _{Q}\left(\frac{1}{|Q|} \int_{Q} w(x)^{q} d x\right)^{1 / q}\left(\frac{1}{|Q|} \int_{Q} w(x)^{-p /(p-1)} d x\right)^{(p-1) / p}<\infty\right\}
\end{gathered}
$$

for $1<p, q<\infty$.

Given a weight function $w$, for $1<p<\infty$, the weighted Lebesgue space $L^{p}(w)$ is the space of functions $f$ such that

$$
\|f\|_{L^{p}(w)}=\left(\int_{R^{n}}|f(x)|^{p} w(x) d x\right)^{1 / p}<\infty .
$$


For $\beta>0$ and $p>1$, let $\dot{F}_{p}^{\beta, \infty}(w)$ be the weighted homogeneous TriebelLizorkin space. For $0<\beta<1$, the weighted Lipschitz space $\operatorname{Lip}_{\beta}(w)$ is the space of functions $f$ such that

$$
\|f\|_{\operatorname{Lip}_{\beta}(w)}=\sup _{Q} \frac{1}{w(Q)^{1+\beta / n}} \int_{Q}\left|f(y)-f_{Q}\right| d y<\infty .
$$

Given some function $b_{j} \in \operatorname{Lip}_{\beta}(w), 1 \leq j \leq m$, we denote by $C_{j}^{m}$ the family of all finite subsets $\sigma=\{\sigma(1), \cdots, \sigma(j)\}$ of $\{1, \cdots, m\}$ of $j$ different elements and $\sigma(i)<\sigma(j)$ when $i<j$. For $\sigma \in C_{j}^{m}$, set $\sigma^{c}=\{1, \cdots, m\} \backslash \sigma$. For $\vec{b}=\left(b_{1}, \cdots, b_{m}\right)$ and $\sigma=\{\sigma(1), \cdots, \sigma(j)\} \in C_{j}^{m}$, set $\vec{b}_{\sigma}=\left(b_{\sigma(1)}, \cdots, b_{\sigma(j)}\right)$, $b_{\sigma}=\prod_{i=1}^{j} b_{\sigma(i)}$ and $\left\|\vec{b}_{\sigma}\right\|_{L i p_{\beta}(w)}=\prod_{i=1}^{j}\left\|b_{\sigma(i)}\right\|_{L i p_{\beta}(w)}$.

Now we shall state our theorems as following.

Theorem 1. Let $b_{j} \in \operatorname{Lip}_{\beta}(w)$ for $1 \leq j \leq m, 0<\beta<1$ and $w \in A_{1}$. Suppose $1<p<n / m \beta$ and $1 / q=1 / p-m \beta / n$. Then $T_{\vec{b}}$ is bounded from $L^{p}(w)$ to $L^{q}\left(w^{1-m+(q-1) m \beta / n}\right)$.

Theorem 2.Let $b_{j} \in \operatorname{Lip}_{\beta}(w)$ for $1 \leq j \leq m, 0<\beta<1$ and $w \in A_{1}$. Suppose $1<p<\infty$. Then $T_{\vec{b}}$ is bounded from $L^{p}(w)$ to $\tilde{F}_{p}^{m \beta, \infty}\left(w^{1-m-m \beta / n}\right)$.

\section{Proofs of Theorems}

In order to prove the theorems, we need the following lemmas.

Lemma 1.(see [11][13]) For $0<\beta<1, w \in A_{1}, b \in \operatorname{Lip}_{\beta}(w)$ and $1 \leq p \leq$ $\infty$, we have

$$
\|b\|_{L i p_{\beta}(w)} \approx \sup _{Q} w(Q)^{-\beta / n}\left(w(Q)^{-1} \int_{Q}\left|b(x)-b_{Q}\right|^{p} w(x)^{1-p} d x\right)^{1 / p} .
$$

Lemma 2.(see [10][13]) For $0<\beta<1, w \in A_{1}, b \in \operatorname{Lip}_{\beta}(w)$ and any cube $Q$, we have

$$
\sup _{x \in Q}\left|b(x)-b_{Q}\right| \leq C|| b||_{\text {Lip }_{\beta}(w)} w(Q)^{1+\beta / n}|Q|^{-1} .
$$

Lemma 3. For $0<\beta<1, w \in A_{1}, b \in \operatorname{Lip}_{\beta}(w)$, any cube $Q$ and $\tilde{x} \in Q$, we have

$$
\left|b_{2^{k} Q}-b_{Q}\right| \leq C k w(\tilde{x}) w\left(2^{k} Q\right)^{\beta / n}|| b \|_{L_{i p_{\beta}}(w)} .
$$


Lemma 4.(see [12]) For $0<\beta<1, w \in A_{1}, 1<p<\infty$ and $m>0$, we have

$$
\begin{aligned}
\|f\|_{\tilde{F}_{p}^{m \beta, \infty}(w)} & \approx\left\|\sup _{Q \ni \tilde{x}}|Q|^{-1-m \beta / n} \int_{Q}\left|f(x)-f_{Q}\right| d x \mid\right\|_{L^{p}(w)} \\
& \approx\left\|\sup _{Q \ni \tilde{x}} \inf _{c \in C}|Q|^{-1-m \beta / n} \int_{Q}|f(x)-c| d x\right\|_{L^{p}(w)}
\end{aligned}
$$

Lemma 5.(see [11]) Suppose that $0 \leq \eta<n, 1<s<p<n / \eta, 1 / q=$ $1 / p-\eta / n$ and $w \in A(p, q)$. Then

$$
\left\|M_{\eta, s}(f)\right\|_{L^{q}\left(w^{q}\right)} \leq C\|f\|_{L^{p}\left(w^{p}\right)} .
$$

Proof of Theorem 1. In order to prove the theorem, we first prove a sharp function estimate for the multilinear commutator. We will prove that for any $1<r<\infty$ and cube $Q$, there exists some constant $C_{0}$ such that

$$
\begin{aligned}
\frac{1}{|Q|} \int_{Q}\left|T_{\vec{b}}(f)(x)-C_{0}\right| d x \leq & C|| \vec{b} \|_{L i p_{\beta}(w)} w(\tilde{x})^{m+m \beta / n}\left(M_{m \beta, r}(f)(\tilde{x})+\right. \\
& \left.M_{m \beta, r}(T(f))(\tilde{x})\right) .
\end{aligned}
$$

Fix a cube $Q=Q\left(x_{0}, d\right)$ and $\tilde{x} \in Q$, we write $f(x)=f_{1}(x)+f_{2}(x)$ and $f_{1}=f \chi_{2 Q}, f_{2}=f \chi_{(2 Q)^{c}}$.

We first consider the Case $m=1$. For $C_{0}=T\left(\left(\left(b_{1}\right)_{2 Q}-b_{1}\right) f_{2}\right)\left(x_{0}\right)$, we write $F_{t}^{b_{1}}(f)(x)=\left(b_{1}(x)-\left(b_{1}\right)_{2 Q}\right) F_{t}(f)(x)-F_{t}\left(\left(b_{1}-\left(b_{1}\right)_{2 Q}\right) f_{1}\right)(x)-F_{t}\left(\left(b_{1}-\right.\right.$ $\left.\left.\left(b_{1}\right)_{2 Q}\right) f_{2}\right)(x)$. Then

$$
\begin{aligned}
& \left|T_{b_{1}}(f)(x)-T\left(\left(\left(b_{1}\right)_{2 Q}-b_{1}\right) f_{2}\right)\left(x_{0}\right)\right| \\
= & \left\|F_{t}^{b_{1}}(f)(x)\right\|-\left\|F_{t}\left(\left(\left(b_{1}\right)_{2 Q}-b_{1}\right) f_{2}\right)\left(x_{0}\right)\right\| \| \\
\leq & \left\|F_{t}^{b_{1}}(f)(x)-F_{t}\left(\left(\left(b_{1}\right)_{2 Q}-b_{1}\right) f_{2}\right)\left(x_{0}\right)\right\| \\
\leq & \left\|\left(b_{1}(x)-\left(b_{1}\right)_{2 Q}\right) F_{t}(f)(x)\right\|+\left\|F_{t}\left(\left(b_{1}-\left(b_{1}\right)_{2 Q}\right) f_{1}\right)(x)\right\| \\
& +\left\|F_{t}\left(\left(b_{1}-\left(b_{1}\right)_{2 Q}\right) f_{2}\right)(x)-F_{t}\left(\left(b_{1}-\left(b_{1}\right)_{2 Q}\right) f_{2}\right)\left(x_{0}\right)\right\| \\
= & A(x)+B(x)+C(x) .
\end{aligned}
$$

For $A(x)$, by Hölder's inequality and lemma 2, we have

$$
\begin{aligned}
& \frac{1}{|Q|} \int_{Q} A(x) d x \\
\leq & \frac{1}{|Q|} \int_{Q}\left|b_{1}(x)-\left(b_{1}\right)_{2 Q}\right||T(f)(x)| d x \\
\leq & \frac{1}{|Q|}\left(\int_{Q}\left|b_{1}(x)-\left(b_{1}\right)_{2 Q}\right|^{r^{\prime}} d x\right)^{\frac{1}{r^{\prime}}}\left(\int_{Q}|T(f)(x)|^{r} d x\right)^{\frac{1}{r}}
\end{aligned}
$$




$$
\begin{aligned}
\leq & \frac{1}{|2 Q|} \sup _{x \in 2 Q}\left|b_{1}(x)-\left(b_{1}\right)_{2 Q}\right||Q|^{\frac{1}{r^{\prime}}}\left(\int_{Q}|T(f)(x)|^{r}(x) d x\right)^{\frac{1}{r}} \\
\leq & \frac{C}{|Q|}|| b_{1} \|_{L^{\prime} p_{\beta}(w)} w(Q)^{1+\beta / n}|Q|^{-1}|Q|^{1 / r^{\prime}}|Q|^{1 / r-\beta / n} \\
& \cdot\left(\frac{1}{|Q|^{1-r \beta / n}} \int_{Q}|T(f)(x)|^{r} d x\right)^{\frac{1}{r}} \\
\leq & C|| b_{1} \|_{L_{i p_{\beta}}(w)}\left(\frac{w(Q)}{|Q|}\right)^{1+\beta / n} M_{\beta, r}(T(f))(\tilde{x}) \\
\leq & C\left\|b_{1}\right\|_{\operatorname{Lip}_{\beta}(w)} w(\tilde{x})^{1+\beta / n} M_{\beta, r}(T(f))(\tilde{x}) .
\end{aligned}
$$

For $B(x)$, by the type $(r, r)$ of $T$ and Lemma 2, we obtain

$$
\begin{aligned}
& \frac{1}{|Q|} \int_{Q} B(x) d x \\
\leq & \frac{1}{|Q|}\left(\int_{R^{n}}\left|T\left(\left(\left(b_{1}\right)_{Q}-b_{1}\right) f_{1}\right)(x)\right|^{r} d x\right)^{\frac{1}{r}}|Q|^{\frac{1}{r^{\prime}}} \\
\leq & \frac{C}{|Q|}\left(\int_{R^{n}}\left|\left(b_{1}\right)_{Q}-b_{1}(x)\right|^{r}\left|f_{1}(x)\right|^{r} d x\right)^{\frac{1}{r}}|Q|^{\frac{1}{r^{\prime}}} \\
\leq & \frac{C}{|Q|} \sup _{x \in 2 Q}\left|b_{1}(x)-\left(b_{1}\right)_{2 Q}\right|\left(\int_{2 Q}|f(x)|^{r} d x\right)^{\frac{1}{r}}|Q|^{\frac{1}{r^{\prime}}} \\
\leq & \left.\frac{C}{|Q|}|| b_{1}\right|_{L i p_{\beta}(w)} w(2 Q)^{1+\beta / n}|2 Q|^{-1}|2 Q|^{1 / r-\beta / n} . \\
& \cdot\left(\frac{1}{|2 Q|^{1-r \beta / n}} \int_{2 Q}|f(x)|^{r} d x\right)^{\frac{1}{r}}|Q|^{\frac{1}{r^{\prime}}} \\
\leq & C|| b_{1} \|_{L_{i p_{\beta}}(w)}\left(\frac{w(2 Q)}{|2 Q|}\right)^{1+\beta / n} M_{\beta, r}(f)(x) \\
\leq & C|| b_{1} \|_{L_{i p_{\beta}}(w)} w(\tilde{x})^{1+\beta / n} M_{\beta, r}(f)(\tilde{x}) .
\end{aligned}
$$


Vol. L (2012) Weighted Lipschitz Estimates for Multilinear...

For $C(x)$, for any $x \in Q$, we have

$$
\begin{aligned}
& C(x)=\left\|\int_{R^{n}}\left(b_{1}(y)-\left(b_{1}\right)_{2 Q}\right) f_{2}(y)\left(F_{t}(x, y)-F_{t}\left(x_{0}, y\right)\right) d y\right\| \\
& \leq \int_{(2 Q)^{c}}\left|b_{1}(y)-\left(b_{1}\right)_{2 Q}\|f(y)\|\right| F_{t}(x, y)-F_{t}\left(x_{0}, y\right)\|\| d y \\
& \leq C \int_{(2 Q)^{c}}\left|b_{1}(y)-\left(b_{1}\right)_{2 Q}\right||f(y)| \frac{\left|x_{0}-x\right|^{\varepsilon}}{\left|x_{0}-y\right|^{n+\varepsilon}} d y \\
& \leq C \sum_{k=1}^{\infty} \int_{2^{k+1} Q \backslash 2^{k} Q}\left|b_{1}(y)-\left(b_{1}\right)_{2 Q} \| f(y)\right| \frac{\left|x_{0}-x\right|^{\varepsilon}}{\left|x_{0}-y\right|^{n+\varepsilon}} d y \\
& \leq C \sum_{k=1}^{\infty} 2^{-k \varepsilon} \frac{1}{\left|2^{k+1} Q\right|} \int_{2^{k+1} Q}\left|b_{1}(y)-\left(b_{1}\right)_{2^{k+1} Q}\right||f(y)| d y \\
& +C \sum_{k=1}^{\infty} 2^{-k \varepsilon} \frac{1}{\left|2^{k+1} Q\right|} \int_{2^{k+1} Q}\left|\left(b_{1}\right)_{2^{k+1} Q}-\left(b_{1}\right)_{Q}\right||f(y)| d y \\
& \leq C \sum_{k=1}^{\infty} 2^{-k \varepsilon} \frac{1}{\left|2^{k+1} Q\right|}\left(\int_{2^{k+1} Q}\left|b_{1}(y)-\left(b_{1}\right)_{2^{k+1} Q}\right|^{r^{\prime}} d y\right)^{\frac{1}{r^{\prime}}} \text {. } \\
& \cdot\left(\int_{2^{k+1} Q}|f(y)|^{r} d y\right)^{\frac{1}{r}}+ \\
& +C \sum_{k=1}^{\infty} 2^{-k \varepsilon} \frac{1}{\left|2^{k+1} Q\right|}\left|\left(b_{1}\right)_{2^{k+1} Q}-\left(b_{1}\right)_{2 Q}\right|\left(\int_{2^{k+1} Q}|f(y)|^{r} d y\right)^{\frac{1}{r}}\left|2^{k+1} Q\right|^{\frac{1}{r^{\prime}}} \\
& \leq C \sum_{k=1}^{\infty} 2^{-k \varepsilon} \sup _{y \in 2^{k+1} Q}\left|b_{1}(y)-\left(b_{1}\right)_{2^{k+1} Q}\right|\left|2^{k+1} Q\right|^{-\beta / n} \text {. } \\
& \cdot\left(\frac{1}{\left|2^{k+1} Q\right|^{1-r \beta / n}} \int_{2^{k+1} Q}|f(y)|^{r} d y\right)^{\frac{1}{r}}+ \\
& +C \sum_{k=1}^{\infty} 2^{-k \varepsilon}\left|\left(b_{1}\right)_{2^{k+1} Q}-\left(b_{1}\right)_{2 Q}\right|\left|2^{k+1} Q\right|^{-\beta / n} . \\
& \cdot\left(\frac{1}{\left|2^{k+1} Q\right|^{1-r \beta / n}} \int_{2^{k+1} Q}|f(y)|^{r} d y\right)^{\frac{1}{r}} \\
& \leq C \sum_{k=1}^{\infty} 2^{-k \varepsilon}|| b_{1} \|_{L_{i p_{\beta}}(w)} w\left(2^{k+1} Q\right)^{1+\beta / n}\left|2^{k+1} Q\right|^{-1}\left|2^{k+1} Q\right|^{-\beta / n} M_{\beta, r}(f)(\tilde{x}) \\
& +C \sum_{k=1}^{\infty} 2^{-k \varepsilon} k w(\tilde{x}) w\left(2^{k} Q\right)^{\beta / n}|| b_{1} \|_{L_{i p_{\beta}}(w)}\left|2^{k+1} Q\right|^{-\beta / n} M_{\beta, r}(f)(\tilde{x}) \\
& \leq C\left\|b_{1}\right\|_{L i p_{\beta}(w)} \sum_{k=1}^{\infty} 2^{-k \varepsilon}\left(\frac{w\left(2^{k+1} Q\right)}{\left|2^{k+1} Q\right|}\right)^{1+\beta / n} M_{\beta, r}(f)(\tilde{x})
\end{aligned}
$$




$$
\begin{aligned}
& +C\left\|b_{1}\right\|_{L_{i p_{\beta}}(w)} \sum_{k=1}^{\infty} k 2^{-k \varepsilon} w(\tilde{x})\left(\frac{w\left(2^{k} Q\right)}{\left|2^{k+1} Q\right|}\right)^{\beta / n} M_{\beta, r}(f)(\tilde{x}) \\
\leq & C\left\|b_{1}\right\|_{L_{i p_{\beta}}(w)} w(\tilde{x})^{1+\beta / n} M_{\beta, r}(f)(\tilde{x}),
\end{aligned}
$$

thus, we can obtain

$$
\frac{1}{|Q|} \int_{Q} C(x) d x \leq C\left\|b_{1}\right\|_{L_{i p_{\beta}}(w)} w(\tilde{x})^{1+\beta / n} M_{\beta, r}(f)(\tilde{x}) .
$$

Now, we consider the Case $m \geq 2$. For $b=\left(b_{1}, \cdots, b_{m}\right)$,we have,

$$
\begin{aligned}
& F_{t}^{\vec{b}}(f)(x)=\int_{R^{n}}\left[\prod_{j=1}^{m}\left(b_{j}(x)-b_{j}(y)\right)\right] F_{t}(x, y) f(y) d y \\
= & \int_{R^{n}} \prod_{j=1}^{m}\left(\left[\left(b_{j}(x)-\left(b_{j}\right)_{2 Q}\right)-\left(b_{j}(y)-\left(b_{j}\right)_{2 Q}\right)\right] F_{t}(x, y) f(y) d y\right. \\
= & \sum_{j=0}^{m} \sum_{\sigma \in C_{j}^{m}}(-1)^{m-j}\left(b(x)-(b)_{2 Q}\right)_{\sigma} \int_{R^{n}}\left(b(y)-(b)_{2 Q}\right)_{\sigma} F_{t}(x, y) f(y) d y \\
= & \left(b_{1}(x)-\left(b_{1}\right)_{2 Q}\right) \cdots\left(b_{m}(x)-\left(b_{m}\right)_{2 Q}\right) F_{t}(f)(x) \\
& +(-1)^{m} F_{t}\left(\left(b_{1}-\left(b_{1}\right)_{2 Q}\right) \cdots\left(b_{m}-\left(b_{m}\right)_{2 Q}\right) f\right)(x) \\
& +\sum_{j=1}^{m-1} \sum_{\sigma \in C_{j}^{m}}(-1)^{m-j}\left(b(x)-(b)_{2 Q}\right)_{\sigma} \int_{R^{n}}(b(y)-b(x))_{\sigma^{c}} F_{t}(x, y) f(y) d y \\
= & \left(b_{1}(x)-\left(b_{1}\right)_{2 Q}\right) \cdots\left(b_{m}(x)-\left(b_{m}\right)_{2 Q}\right) F_{t}(f)(x) \\
& +(-1)^{m} F_{t}\left(\left(b_{1}-\left(b_{1}\right)_{2 Q}\right) \cdots\left(b_{m}-\left(b_{m}\right)_{2 Q}\right) f\right)(x) \\
& +\sum_{j=1}^{m-1} \sum_{\sigma \in C_{j}^{m}}(-1)^{m-j}\left(b(x)-(b)_{2 Q}\right)_{\sigma} F_{t}^{\vec{b}_{\sigma^{c}}}(f)(x)
\end{aligned}
$$

thus

$$
\begin{aligned}
& \left.\mid T_{\vec{b}}(f)(x)-T\left(\left(\left(b_{1}\right)_{2 Q}-b_{1}\right) \cdots\left(\left(b_{m}\right)_{2 Q}-b_{m}\right)\right) f_{2}\right)\left(x_{0}\right) \mid \\
= & ||\left|F_{t}^{\vec{b}}(f)(x)\|-\| F_{t}\left(\left(\left(b_{1}\right)_{2 Q}-b_{1}\right) \cdots\left(\left(b_{m}\right) 2 Q-b_{m}\right) f_{2}\right)\left(x_{0}\right) \|\right| \mid
\end{aligned}
$$


Vol. L (2012) Weighted Lipschitz Estimates for Multilinear...

$$
\begin{aligned}
& \leq\left\|F_{t}^{\vec{b}}(f)(x)-F_{t}\left(\left(\left(b_{1}\right)_{2 Q}-b_{1}\right) \cdots\left(\left(b_{m}\right)_{2 Q}-b_{m}\right) f_{2}\right)\left(x_{0}\right)\right\| \\
& \leq\left\|\left(b_{1}(x)-\left(b_{1}\right)_{2 Q}\right) \cdots\left(b_{m}(x)-\left(b_{m}\right)_{2 Q}\right) F_{t}(f)(x)\right\| \\
& +\sum_{j=1}^{m-1} \sum_{\sigma \in C_{j}^{m}}\left\|\left(\vec{b}(x)-\left(b_{m}\right)_{2 Q}\right)_{\sigma} F_{t}^{\vec{b}_{\sigma} c}(f)(x)\right\| \\
& +\left\|F_{t}\left(\left(b_{1}-\left(b_{1}\right)_{2 Q}\right) \cdots\left(b_{m}-\left(b_{m}\right)_{2 Q}\right) f_{1}\right)(x)\right\| \\
& +\| F_{t}\left(\prod_{j=1}^{m}\left(\left(b_{j}-\left(b_{j}\right)_{2 Q}\right) f_{2}\right)(x)-F_{t}\left(\prod_{j=1}^{m}\left(b_{j}-\left(b_{j}\right)_{2 Q}\right) f_{2}\right)\left(x_{0}\right) \|\right. \\
& =A_{1}(x)+B_{1}(x)+C_{1}(x)+D_{1}(x) .
\end{aligned}
$$

For $A_{1}(x)$, by Hölder's inequality and lemma 2, we get,

$$
\begin{aligned}
& \frac{1}{|Q|} \int_{Q} A_{1}(x) d \mu(x) \\
\leq & \frac{1}{|Q|} \int_{Q}\left|\prod_{j=1}^{m}\left(b_{j}(x)-\left(b_{j}\right)_{2 Q}\right)\right||T(f)(x)| d x \\
\leq & \frac{1}{|2 Q|} \prod_{j=1}^{m}\left(\int_{2 Q}\left|b_{j}(x)-\left(b_{j}\right)_{2 Q}\right|^{r_{j}} d x\right)^{\frac{1}{r_{j}}}\left(\int_{Q}|T(f)(x)|^{r} d x\right)^{\frac{1}{r}} \\
\leq & \frac{1}{|2 Q|} \prod_{j=1}^{m}\left(\sup _{x \in 2 Q}\left|b_{j}(x)-\left(b_{j}\right)_{2 Q}\right||2 Q|^{\frac{1}{r_{j}}}\right)\left(\int_{Q}|T(f)(x)|^{r} d x\right)^{\frac{1}{r}} \\
\leq & C \frac{1}{|Q|} \prod_{j=1}^{m}\left(\| b_{j}||_{L i p_{\beta}(w)} w(2 Q)^{1+\beta / n}|2 Q|^{-1}\right)|Q|^{\left(1-\frac{1}{r}\right)+\left(\frac{1}{r}-m \beta / n\right)} . \\
\leq & C\left(\frac{1}{|Q|^{1-r m \beta / n}} \int_{Q}|T(f)(x)|^{r} d x\right)^{\frac{1}{r}} \\
\leq & C \|\left.\vec{b}\right|_{L i p_{\beta}(w)} w(2 Q)^{m+m \beta / n}|2 Q|^{-m-m \beta / n} M_{m \beta, r}(T(f))(\tilde{x}) \\
\leq & C|| \vec{b} \|_{L_{L i p_{\beta}(w)} w(\tilde{x})^{m+m \beta / n} M_{m \beta, r}(T(f))(\tilde{x}) .}
\end{aligned}
$$


For $B_{1}(x)$, similar to $B(x)$, using the boundness of $T$ and Lemma 2 , we get

$$
\begin{aligned}
& \frac{1}{|Q|} \int_{Q} B_{1}(x) d x \\
\leq & \frac{1}{|Q|}\left(\int_{R^{n}}\left|T\left(\prod_{j=1}^{m}\left(b_{j}-\left(b_{j}\right)_{2 Q}\right) f_{1}\right)(x)\right|^{r} d x\right)^{\frac{1}{r}}|Q|^{\frac{1}{r^{\prime}}} \\
\leq & \frac{C}{|Q|}\left(\int_{R^{n}}\left|\prod_{j=1}^{m}\left(b_{j}(x)-\left(b_{j}\right)_{2 Q}\right) f_{1}(x)\right|^{r} d x\right)^{\frac{1}{r}}|Q|^{\frac{1}{r^{\prime}}} \\
\leq & \frac{C}{|Q|} \prod_{j=1}^{m} \sup _{x \in 2 Q}\left|b_{j}(x)-\left(b_{j}\right)_{2 Q}\right|\left(\int_{2 Q}|f(x)|^{r} d x\right)^{\frac{1}{r}}|Q|^{\frac{1}{r^{\prime}}} \\
\leq & \frac{C}{|Q|}\left(\prod_{j=1}^{m}|| b_{j}||_{L i p_{\beta}(w)} w(2 Q)^{1+\beta / n}|2 Q|^{-1}\right)|2 Q|^{\frac{1}{r^{\prime}}+\frac{1}{r}-m \beta / n} . \\
\leq & \cdot\left(\frac{1}{|2 Q|^{1-r m \beta / n}} \int_{2 Q}|f(x)|^{r} d x\right)^{\frac{1}{r}} \\
\leq & C|| \vec{b} \|_{L_{L i p_{\beta}}(w)}\left(\frac{w(2 Q)}{\mid 2 Q \|_{p_{\beta}(w)} w(\tilde{x})^{m+m \beta / n} M_{m \beta, r}(f)(\tilde{x}) .}\right.
\end{aligned}
$$

For $C_{1}(x)$, by Hölder's inequality and lemma 2 , we get

$$
\begin{aligned}
& \frac{1}{|Q|} \int_{Q} C_{1}(x) d x \\
\leq & C \sum_{j=1}^{m-1} \sum_{\sigma \in C_{j}^{m}} \frac{1}{|2 Q|} \int_{2 Q}\left|\left(b_{j}(x)-\left(b_{j}\right)_{2 Q}\right)_{\sigma}\right|\left|T\left(\left(\left(b_{j}-\left(b_{j}\right)_{2 Q}\right)_{\sigma^{c}}\right) f\right)(x)\right| d x \\
\leq & C \sum_{j=1}^{m-1} \sum_{\sigma \in C_{j}^{m}} \frac{1}{|2 Q|}\left(\int_{2 Q}\left|\left(b_{j}(x)-\left(b_{j}\right)_{2 Q}\right)_{\sigma}\right|^{r^{\prime}} d x\right)^{\frac{1}{r^{\prime}}} \cdot \\
\leq & \left.\left.C \sum_{j=1}^{m-1} \sum_{\sigma \in C_{j}^{m}} \frac{1}{|2 Q|} \sup _{x \in 2 Q}\left|\left(\left(b_{j}(x)-\left(b_{j}-\left(b_{j}\right)_{2 Q}\right)_{\sigma^{c}}\right) f\right)(x)\right|_{\sigma}\right|^{r} d x\right)\left.^{\frac{1}{r}}|| 2 Q\right|^{\frac{1}{r^{\prime}}} \sup _{x \in 2 Q}\left|\left(b_{j}(x)-\left(b_{j}\right)_{2 Q}\right)_{\sigma^{c}}\right| . \\
& \cdot\left(\int_{2 Q}|T(f)(x)|^{r} d x\right)^{\frac{1}{r}}
\end{aligned}
$$




$$
\begin{aligned}
\leq & C \sum_{j=1}^{m-1} \sum_{\sigma \in C_{j}^{m}} \frac{1}{|2 Q|}\left\|\overrightarrow{b_{\sigma}}\right\|_{\text {Lip }_{\beta}(w)} w(2 Q)^{j+j \beta / n}|2 Q|^{-j}\left\|\overrightarrow{b_{\sigma^{c}}}\right\|_{L_{i_{\beta}}(w)} \\
& \cdot w(2 Q)^{(m-j)+(m-j) \beta / n}|2 Q|^{\frac{1}{r^{\prime}}+\frac{1}{r}-m \beta / n-(m-j)} \\
& \cdot\left(\frac{1}{|2 Q|^{1-r m \beta / n}} \int_{2 Q}|T(f)(x)|^{r} d x\right)^{\frac{1}{r}} \\
\leq & C \|\left.\vec{b}\right|_{\operatorname{Lip}_{\beta}(w)}\left(\frac{w(2 Q)}{|2 Q|}\right)^{m+m \beta / n} M_{m \beta, r}(T(f))(\tilde{x}) \\
\leq & C\|\vec{b}\|_{\operatorname{Lip}_{\beta}(w)} w(\tilde{x})^{m+m \beta / n} M_{m \beta, r}(T(f))(\tilde{x}) .
\end{aligned}
$$

For $D_{1}(x)$, similar to the methods above, we have

$$
\begin{aligned}
D_{1}(x)= & || F_{t}\left(\left(b_{1}-\left(b_{1}\right)_{2 Q}\right) \cdots\left(b_{m}-\left(b_{m}\right)_{2 Q}\right) f_{2}\right)(x)-F_{t}\left(\left(b_{1}-\left(b_{1}\right)_{2 Q}\right) \cdots\right. \\
& \left.\left(b_{m}-\left(b_{m}\right)_{2 Q}\right) f_{2}\right)\left(x_{0}\right)|| \\
\leq & \int_{R^{n}}\left|\prod_{j=1}^{m}\left(b_{j}(y)-b\left(b_{j}\right)_{2 Q}\right)\right|\left|f(y) \chi_{(2 Q)^{c}}(y)\right||| F_{t}(x, y)-F_{t}\left(x_{0}, y\right) \| d y \\
\leq & C \int_{(2 Q)^{c}}\left|\prod_{j=1}^{m}\left(b_{j}(y)-\left(b_{j}\right)_{2 Q}\right)\right||f(y)| \frac{\left|x-x_{0}\right|^{\varepsilon}}{\left|x_{0}-y\right|^{n+\varepsilon}} d y \\
\leq & C \sum_{k=1}^{\infty} \int_{2^{k+1} Q \backslash 2^{k} Q}\left|x-x_{0}\right|^{\varepsilon}\left|x_{0}-y\right|^{-(n+\varepsilon)}\left|\prod_{j=1}^{m}\left(b_{j}(y)-\left(b_{j}\right)_{2 Q}\right)\right||f(y)| d y \\
\leq & C \sum_{k=1}^{\infty} 2^{-k \varepsilon} \frac{1}{\left|2^{k} Q\right|} \\
& \int_{2^{k+1} Q}\left|\prod_{j=1}^{m}\left(\left(b_{j}(y)-\left(b_{j}\right)_{2^{k+1} Q}\right)+\left(\left(b_{j}\right)_{2^{k+1} Q}-\left(b_{j}\right)_{2 Q}\right)\right)\right||f(y)| d y \\
\leq & C \sum_{k=1}^{\infty} 2^{-k \varepsilon} \frac{1}{\left|2^{k} Q\right|} \prod_{j=1}^{m}\left[\int_{2^{k+1} Q}\left|\left(b_{j}(y)-\left(b_{j}\right)_{2^{k+1} Q}\right)\right||f(y)| d y\right. \\
& \left.+\left|\left(b_{j}\right)_{2^{k+1} Q}-\left(b_{j}\right)_{2 Q}\right| \int_{2^{k+1} Q}|f(y)| d y\right]
\end{aligned}
$$




$$
\begin{array}{rl}
\leq & C \sum_{k=1}^{\infty} 2^{-k \varepsilon} \frac{1}{\left|2^{k} Q\right|} \prod_{j=1}^{m}\left[\left(\int_{2^{k+1} Q}\left|\left(b_{j}(y)-\left(b_{j}\right)_{2^{k+1} Q}\right)_{\sigma^{c}}\right|^{r^{\prime}} d y\right)^{\frac{1}{r^{\prime}}}\left(\int_{2^{k+1} Q}|f(y)|^{r} d y\right)^{\frac{1}{r}}\right. \\
& \left.+\left|\left(\left(b_{j}\right)_{2^{k+1} Q}-\left(b_{j}\right)_{2 Q}\right)\right|\right]\left|2^{k+1} Q\right|^{\frac{1}{r^{\prime}}+\left(\frac{1}{r}-m \beta / n\right)}\left(\frac{1}{\left|2^{k+1} Q\right|^{1-r m \beta / n}} \int_{2^{k+1} Q}|f(y)|^{r} d y\right)^{\frac{1}{r}} \\
\leq & C \| \vec{b}||_{L i p_{\beta}(w)} w(\tilde{x})^{j}\left(\frac{w\left(2^{k+1} Q\right)}{\left|2^{k+1} Q\right|}\right)^{(m-j)+m \beta / n} M_{m \beta, r}(f)(\tilde{x}) \\
\leq C & C \|\left.\vec{b}\right|_{L_{\text {Lip }}(w)} w(\tilde{x})^{m+m \beta / n} M_{m \beta, r}(f)(\tilde{x}) .
\end{array}
$$

Thus, we get

$$
\frac{1}{|Q|} \int_{Q} D_{1}(x) d x \leq C|| \vec{b} \|_{L_{i p_{\beta}(w)} w(\tilde{x})^{m+m \beta / n} M_{m \beta, r}(f)(\tilde{x}) .}
$$

Combining all the estimates above, we get

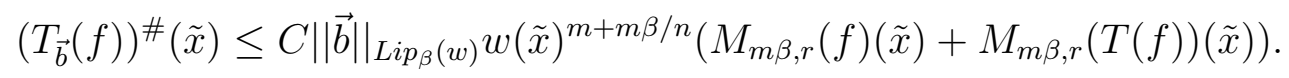

Now, choose $1<r<p$ and by lemma 5 , we have

$$
\begin{aligned}
& \left\|T_{\vec{b}}(f)\right\|_{L^{q}\left(w^{1-m+(q-1) m \beta / n}\right)} \\
\leq & C\left\|M\left(T_{\vec{b}}(f)\right)\right\|_{L^{q}\left(w^{1-m+(q-1) m \beta / n}\right)} \\
\leq & C\left\|\left(T_{\vec{b}}(f)\right)^{\#}\right\|_{L^{q}\left(w^{1-m+(q-1) m \beta / n}\right)} \\
\leq & C\|\vec{b}\|_{L_{i p}(w)}\left(\left\|w^{m+m \beta / n} M_{m \beta, r}(f)\right\|_{L^{q}\left(w^{1-m+(q-1) m \beta / n}\right)}\right. \\
& \left.+\left\|w^{m+m \beta / n} M_{m \beta, r}(T(f))\right\|_{L^{q}\left(w^{1-m+(q-1) m \beta / n}\right)}\right) \\
\leq & C\|\vec{b}\|_{L_{i_{\beta}}(w)}\left(\left\|M_{m \beta, r}(f)\right\|_{L^{q}\left(w^{\frac{q}{p}}\right)}+\left\|M_{m \beta, r}(T(f))\right\|_{L^{q}\left(w^{\frac{q}{p}}\right)}\right) \\
\leq & C\|\vec{b}\|_{L_{p_{\beta}}(w)}\left(\|f\|_{L^{p}(w)}+\|T(f)\|_{L^{p}(w)}\right) \\
\leq & C\|\vec{b}\|_{L_{i p_{\beta}(w)}}\|f\|_{L^{p}(w) .}
\end{aligned}
$$

This completes the proof of Theorem 1.

Proof of Theorem 2. Similar to Theorem 1, for any $1<r<\infty$ and cube $Q$, there exists some constant $C_{0}$ such that for any $\tilde{x} \in Q$,

$$
\frac{1}{|Q|^{1+m \beta / n}} \int_{Q}\left|T_{\vec{b}}(f)(x)-C_{0}\right| d \tilde{x} \leq\left. C|| \vec{b}\right|_{L i p_{\beta}(w)} w(\tilde{x})^{m+m \beta / n}\left(M_{r}(f)(\tilde{x})+M_{r}(T(f))(\tilde{x})\right) \text {. }
$$

Further, we have

$\sup _{\tilde{x} \in Q} \inf _{c \in C} \frac{1}{|Q|^{1+m \beta / n}} \int_{Q}\left|T_{\vec{b}}(x)-c\right| d x \leq C|| \vec{b}||_{L_{i p_{\beta}(w)}} w(\tilde{x})^{m+m \beta / n}\left(M_{r}(f)(\tilde{x})+M_{r}(T(f))(\tilde{x})\right)$. 
Choose $1<r<p$ and by lemma 4 , we obtain

$$
\begin{aligned}
& \left\|T_{\vec{b}}(f)\right\|_{\dot{F}_{p}^{m \beta, \infty}\left(w^{1-m-m \beta / n}\right)} \approx\left\|\sup _{\tilde{x} \in Q} \inf _{c \in C}|Q|^{-1-m \beta / n} \int_{Q}\left|T_{\vec{b}}(f)(x)-c\right| d x\right\|_{L^{p}\left(w^{1-m-m \beta / n}\right)} \\
\leq & C\|\vec{b}\|_{L i p_{\beta}(w)}\left(\left\|w^{m+m \beta / n} M_{r}(f)\right\|_{L^{p}\left(w^{1-m-m \beta / n}\right)}+\left\|w^{m+m \beta / n} M_{r}(T(f))\right\|_{L^{p}\left(w^{1-m-m \beta / n}\right)}\right) \\
\leq & C\|\vec{b}\|_{L i p_{\beta}(w)}\left(\left\|M_{r}(f)\right\|_{L^{p}(w)}+\left\|M_{r}(T(f))\right\|_{L^{p}(w)}\right) \\
\leq & C\|\vec{b}\|_{L_{p_{\beta}}(w)}\left(\|f\|_{L^{p}(w)}+\|T(f)\|_{L^{p}(w)}\right) \\
\leq & C\|\vec{b}\|_{L_{i p_{\beta}(w)}\|f\|_{L^{p}(w)} .}
\end{aligned}
$$

This completes the proof of Theorem 2 .

\section{Applications}

Now we give some applications of the theorems in this paper.

Application 1. Littlewood-Paley operator.

Fixed $\varepsilon>0$. Let $\psi$ be a fixed function which satisfies the following properties:

(1) $\int_{R^{n}} \psi(x) d x=0$,

(2) $|\psi(x)| \leq C(1+|x|)^{-(n+1)}$

(3) $|\psi(x+y)-\psi(x)| \leq C|y|^{\varepsilon}(1+|x|)^{-(n+1+\varepsilon)}$ when $2|y|<|x|$.

The Littlewood-Paley multilinear commutator is defined by

$$
g_{\psi}^{\vec{b}}(f)(x)=\left(\int_{0}^{\infty}\left|F_{t}^{\vec{b}}(f)(x)\right|^{2} \frac{d t}{t}\right)^{1 / 2},
$$

where

$$
F_{t}^{\vec{b}}(f)(x)=\int_{R^{n}} \prod_{j=1}^{m}\left(b_{j}(x)-b_{j}(y)\right) \psi_{t}(x-y) f(y) d y
$$

and $\psi_{t}(x)=t^{-n} \psi(x / t)$ for $t>0$. Set $F_{t}(f)(y)=f * \psi_{t}(y)$. We also define that

$$
g_{\psi}(f)(x)=\left(\int_{0}^{\infty}\left|F_{t}(f)(x)\right|^{2} \frac{d t}{t}\right)^{1 / 2},
$$

which is the Littlewood-Paley operator(see [23]). Let $H$ be the space

$$
H=\left\{h:\|h\|=\left(\int_{0}^{\infty}|h(t)|^{2} d t / t\right)^{1 / 2}<\infty\right\},
$$


then, for each fixed $x \in R^{n}, F_{t}^{\vec{b}}(f)(x)$ may be viewed as the mappings from $[0,+\infty)$ to $H$, and it is clear that

$$
g_{\psi}^{\vec{b}}(f)(x)=\left\|F_{t}^{\vec{b}}(f)(x)\right\|, \quad g_{\psi}(f)(x)=\left\|F_{t}(f)(x)\right\| .
$$

It is easily to see that $g_{\psi}$ satisfies the conditions of Theorems 1 and 2 (see [11-13]), thus Theorems 1 and 2 hold for $g_{\psi}^{\vec{b}}$.

Application 2. Marcinkiewicz operator.

Fixed $0<\gamma \leq 1$. Let $\Omega$ be homogeneous of degree zero on $R^{n}$ with $\int_{S^{n-1}} \Omega\left(x^{\prime}\right) d \sigma\left(x^{\prime}\right)=0$. Assume that $\Omega \in \operatorname{Lip}_{\gamma}\left(S^{n-1}\right)$. The Marcinkiewicz multilinear commutator is defined by

$$
\mu_{\Omega}^{\vec{b}}(f)(x)=\left(\int_{0}^{\infty}\left|F_{t}^{\vec{b}}(f)(x)\right|^{2} \frac{d t}{t^{3}}\right)^{1 / 2}
$$

where

$$
F_{t}^{\vec{b}}(f)(x)=\int_{|x-y| \leq t} \prod_{j=1}^{m}\left(b_{j}(x)-b_{j}(y)\right) \frac{\Omega(x-y)}{|x-y|^{n-1}} f(y) d y
$$

Set

$$
F_{t}(f)(x)=\int_{|x-y| \leq t} \frac{\Omega(x-y)}{|x-y|^{n-1}} f(y) d y
$$

We also define that

$$
\mu_{\Omega}(f)(x)=\left(\int_{0}^{\infty}\left|F_{t}(f)(x)\right|^{2} \frac{d t}{t^{3}}\right)^{1 / 2},
$$

which is the Marcinkiewicz operator(see [24]). Let $H$ be the space

$$
H=\left\{h:\|h\|=\left(\int_{0}^{\infty}|h(t)|^{2} d t / t^{3}\right)^{1 / 2}<\infty\right\} .
$$

Then, it is clear that

$$
\mu_{\Omega}^{\vec{b}}(f)(x)=\left\|F_{t}^{\vec{b}}(f)(x)\right\|, \quad \mu_{\Omega}(f)(x)=\left\|F_{t}(f)(x)\right\| .
$$

It is easily to see that $\mu_{\Omega}$ satisfies the conditions of Theorems 1 and 2 (see [14][24]), thus Theorems 1 and 2 hold for $\mu_{\Omega}^{\vec{b}}$.

Application 3. Bochner-Riesz operator.

Let $\delta>(n-1) / 2, B_{t}^{\delta}(f) \hat{)}(\xi)=\left(1-t^{2}|\xi|^{2}\right)_{+}^{\delta} \hat{f}(\xi)$ and $B_{t}^{\delta}(z)=t^{-n} B^{\delta}(z / t)$ for $t>0$. Set

$$
F_{\delta, t}^{\vec{b}}(f)(x)=\int_{R^{n}} \prod_{j=1}^{m}\left(b_{j}(x)-b_{j}(y)\right) B_{t}^{\delta}(x-y) f(y) d y .
$$


The maximal Bochner-Riesz multilinear commutator is defined by

$$
B_{\delta, *}^{\vec{b}}(f)(x)=\sup _{t>0}\left|B_{\delta, t}^{\vec{b}}(f)(x)\right| .
$$

We also define that

$$
B_{\delta, *}(f)(x)=\sup _{t>0}\left|B_{t}^{\delta}(f)(x)\right|
$$

which is the maximal Bochner-Riesz operator(see [17]). Let $H$ be the space $H=\left\{h:|| h||=\sup _{t>0}|h(t)|<\infty\right\}$, then

$$
B_{\delta, *}^{\vec{b}}(f)(x)=\left\|B_{\delta, t}^{A}(f)(x)\right\|, \quad B_{*}^{\delta}(f)(x)=\left\|B_{t}^{\delta}(f)(x)\right\| .
$$

It is easily to see that $B_{\delta, *}^{\vec{b}}$ satisfies the conditions of Theorems 1 and 2 (see [16][25]), thus Theorems 1 and 2 hold for $B_{\delta, *}^{\vec{b}}$.

\section{References}

[1] J. Alvarez, R. J. Babgy, D. S. Kurtz, and C. Pérez, Weighted estimates for commutators of linear operators, Studia Math., 104, (1993), 195-209.

[2] S. Bloom, A commutator theorem and weighted BMO, Trans. Amer. Math. Soc., 292, (1985), 103-122.

[3] H. Q Bui, Characterizations of weighted Besov and Triebel-Lizorkin spaces via temperatures, J. Func. Anal., 55, (1984), 39-62.

[4] S. Chanillo, A note on commutators, Indiana Univ. Math. J., 31, (1982), 7-16.

[5] W. G. Chen, Besov estimates for a class of multilinear singular integrals, Acta Math. Sinica, 16, (2000), 613-626.

[6] R. R. Coifman, R. Rochberg, and G. Weiss, Fractorization theorems for Hardy spaces in several variables, Ann. of Math., 103, (1976), 611-635.

[7] J. Garcia-Cuerva, Weighted $H^{p}$ spaces, Dissert. Math., 162, 1979.

[8] J. Garcia-Cuerva and J. L. Rubio de Francia, Weighted norm inequalities and related topics, North-Holland Math., Amsterdam, 116, 1985.

[9] B. Hu and J. Gu, Necessary and sufficient conditions for boundedness of some commutators with weighted Lipschitz spaces, J. of Math. Anal. and Appl., 340, (2008), 598-605.

[10] S. Janson, Mean oscillation and commutators of singular integral operators, Ark. Math., 16, (1978), 263-270.

[11] L. Z. Liu, Weighted weak type estimates for commutators of Littlewood-Paley operator, Japanese J. of Math., 29(1), (2003), 1-13. 
[12] L. Z. Liu, A sharp endpoint estimate for multilinear Littlewood-Paley operator, Georgian Math. J., 11, (2004), 361-370.

[13] L. Z. Liu, Sharp endpoint inequality for multilinear Littlewood-Paley operator, Kodai Math. J., 27, (2004), 134-143.

[14] L. Z. Liu, A sharp estimate for multilinear Marcinkiewicz integral operator, The Asian J. of Math., 9(2), (2005), 177-184.

[15] L. Z. Liu, Interior estimates in Morrey spaces for solutions of elliptic equations and weighted boundedness for commutators of singular integral operators, Acta Math. Scientia, 25(B)(1), (2005), 89-94.

[16] L. Z. Liu and S. Z. Lu, Weighted weak type inequalities for maximal commutators of Bochner-Riesz operator, Hokkaido Math. J., 32(1), (2003), 85-99.

[17] S. Z. Lu, Four Lectures on Real $H^{p}$ Spaces, World Scientific, River Edge, NI, 1995.

[18] T. Mizuhara, Boundedness of some classical operators on generalized Morrey spaces, in "Harmonic Analysis", Proceedings of a conference held in Sendai, Japan, (1990), 183-189.

[19] B. Muckenhoupt and R. L. Wheeden, Weighted norm inequalities for fractional integral, Trans. Amer. Math. Soc., 192, (1974), 261-274.

[20] M. Paluszynski, Characterization of the Besov spaces via the commutator operator of Coifman, Rochberg and Weiss, 44, (1995), 1-17.

[21] C. Pérez, Endpoint estimate for commutators of singular integral operators, J. Func. Anal., 128, (1995), 163-185.

[22] C. Pérez and R. Trujillo-Gonzalez, Sharp weighted estimates for multilinear commutators, J. London Math. Soc., 65, (2002), 672-692.

[23] E. M. Stein, Harmonic Analysis: real variable methods, orthogonality and oscillatory integrals, Princeton Univ. Press, Princeton NJ, 1993.

[24] A. Torchinsky and S. Wang, A note on the Marcinkiewicz integral, Colloq. Math., 60/61, (1990), 235-24.

[25] B. S. Wu and L. Z. Liu, A sharp estimate for multilinear Bochner-Riesz operator, Studia Sci. Math. Hungarica, 40(1), (2004), 47-59.

Du Jing

Department of Mathematics

Changsha University of Science and Technology

Changsha, 410077

P. R. of China

Huang Chuangxia

Department of Mathematics

Changsha University of Science and Technology

Changsha, 410077

P. R. of China 
Vol. L (2012) Weighted Lipschitz Estimates for Multilinear...

Liu Lanzhe

Department of Mathematics

Changsha University of Science and Technology

Changsha, 410077

P. R. of China

E-mail: lanzheliu@163.com

Received: 10.01.2012

Accepted: 25.05.2012 